\title{
A minimal mathematical model of nonphotochemical quenching of chlorophyll fluorescence
}

\author{
Oliver Ebenhöh ${ }^{\mathrm{a}, \mathrm{b}}$, Torsten Houwaart ${ }^{\mathrm{c}}$, Heiko Lokstein ${ }^{\mathrm{d}}$, Stephanie \\ Schlede $^{\mathrm{d}}$, Katrin Tirok ${ }^{\mathrm{c}, \mathrm{d}}$ \\ ${ }^{a}$ Institute of Complex Systems and Mathematical Biology, SUPA, University of Aberdeen, \\ Meston Walk, Aberdeen AB24 3UE, United Kingdom \\ ${ }^{b}$ Institute of Medical Sciences, University of Aberdeen, Foresterhill, Aberdeen AB25 2ZD, \\ United Kingdom \\ ${ }^{c}$ Max Planck Institute of Molecular Plant Physiology, Am Mühlenberg 1, D-14476 \\ Potsdam-Golm, Germany \\ ${ }^{d}$ Institut für Biochemie und Biologie, Universität Potsdam, Karl-Liebknecht-Str. 24-25, \\ D-14476 Potsdam-Golm, Germany
}

\begin{abstract}
Under natural conditions, plants are exposed to rapidly changing light intensities. To acclimate to such fluctuations, plants have evolved adaptive mechanisms that optimally exploit available light energy and simultaneously minimise damage of the photosynthetic apparatus through excess light. An important mechanism is the dissipation of excess excitation energy as heat which can be measured as nonphotochemical quenching of chlorophyll fluorescence (NPQ). In this paper, we present a highly simplified mathematical model that captures essential experimentally observed features of the short term adaptive quenching dynamics. We investigate the stationary and dynamic behaviour of the model and systematically analyse the dependence of characteristic system properties on key parameters such as rate constants and pool sizes. Comparing simulations with experimental data allows to derive conclusions about the validity of the simplifying assumptions and we further propose hypotheses regarding the role of the xanthophyll cycle in NPQ. We envisage that the presented theoretical description of the light reactions in conjunction with short term adaptive processes serves as a basis for the development of more detailed mechanistic models by which the molecular mechanisms of NPQ can be theoretically studied.
\end{abstract}

Email address: ebenhoeh@abdn.ac.uk (Oliver Ebenhöh)

Preprint submitted to BioSystems

May 25, 2011 
Keywords: photosynthesis, light reactions, nonphotochemical quenching of chlorophyll fluorescence, chlorophyll fluorescence, mathematical model

\section{Introduction}

In oxygenic photosynthesis, plants harvest solar energy and transform it into biochemically useful energy and redox equivalents in the form of ATP and NADPH, which are required to assimilate carbon and nitrogen, and to drive other metabolic and cellular processes. Since under natural environmental conditions, light intensity may vary considerably in time, for example by clouds obscuring the sun, the photosynthetic machinery has to acclimate rapidly to these fluctuations to prevent excess excitation, which may severely damage the photosynthetic apparatus. The evolved mechanisms to optimally exploit the available light energy while simultaneously minimising photodamage include the dissipation of excess excitation energy as heat. This process can be readily measured as nonphotochemical quenching of chlorophyll fluorescence (NPQ) (for a review, see Horton et al., 2008). Three processes contribute to NPQ, termed energy dependent, state transition-related and photo-inhibitory quenching ( $\mathrm{qE}, \mathrm{qT}$, and $\mathrm{qI}$, respectively, see Quick and Stitt 1989; Horton et al. 2008). Under high light conditions, qE presents the major component of NPQ. Several factors are believed to contribute to qE, the interplay of which has not yet been fully elucidated. Energy dependent ( $\mathrm{qE}$ ) quenching is triggered by a high $\Delta \mathrm{pH}$ over the thylakoid membrane and is evidently linked to the xanthophyll cycle (Horton et al., 2008), in which zeaxanthin is formed by enzymatic de-epoxidation of violaxanthin. How exactly zeaxanthin triggers fluorescence quenching is not yet fully clarified, however, it is argued that zeaxanthin may act as a direct quencher (Frank et al., 1994).

Existing mathematical models of chlorophyll fluorescence considering the fast fluorescence induction kinetics on the timescale of microseconds (Zhu et al., 2005; Vredenberg, 2009) are very useful to explore the mechanisms within photosystem II (PSII) which give rise to fluorescence signals. Other models are highly elaborate, encompassing detailed descriptions of the electron transport processes in conjunction with the carbon fixating dark reactions of the Calvin cycle (Laisk et al., 1997, 2006). These approaches are helpful to evaluate whether our general understanding of the entirety of the photosynthetic processes is basically correct. In this paper, we concentrate 
on the particular process of NPQ. For this, we focus on time scales of seconds to minutes, in which the state of the reaction centres (RCs) can be approximated as stationary. We follow the reductionist approach first applied to photosynthesis by Thornley (1974), and deliberately develop a minimal model which is capable of reproducing key biological properties. A minimal model allows for a systematic analysis of the importance of parameters on the overall performance and provides the advantage that unknown parameters can easily be fitted, avoiding the danger of over-fitting. Further, it is partly analytically tractable and allows to understand which basic mechanisms give rise to the system's behaviour. In particular, we explore to what extent experimental data can be reproduced by the simplifying assumption that zeaxanthin directly acts as a quencher. Both, agreement and discrepancies between simulation and data allow for hypotheses on the molecular nature of the quenching processes. Further, based on our analysis, we put forth suggestions for new experiments and more sophisticated models which may further clarify molecular aspects of NPQ.

\section{Model}

NPQ processes are associated with PSII. The observed quenching dynamics take place on the order of seconds to minutes. These two basic observations of NPQ motivate the following simplifying assumptions of our model: a) we only consider processes in PSII; b) We approximate the fast dynamics of charge separation and oxygen evolution by a quasi steady-state approximation. The latter approximation is reasonable because charge separation and oxygen evolution occur on time scales of milliseconds and faster and, since our model aims to simulate the quenching dynamics, the fast processes can be assumed to be in a stationary state on the time scale of seconds and slower.

The model is kept as simple as possible, the basic scheme is given in Fig. 1. Light absorption leads to excitation of chlorophyll molecules in the light harvesting complexes (LHCs). This excitation energy is subsequently transferred to chlorophylls associated with the RC, where it is used to separate charges, leading to a positively charged donor and a negatively charged acceptor. NPQ inhibits excitation energy transfer and allows dissipation of the excitation energy as heat. In the model, light harvesting, excitation energy transfer and charge separation are described by a single transition from state $A_{1}$, representing open RCs, to state $A_{2}$, representing a state with 


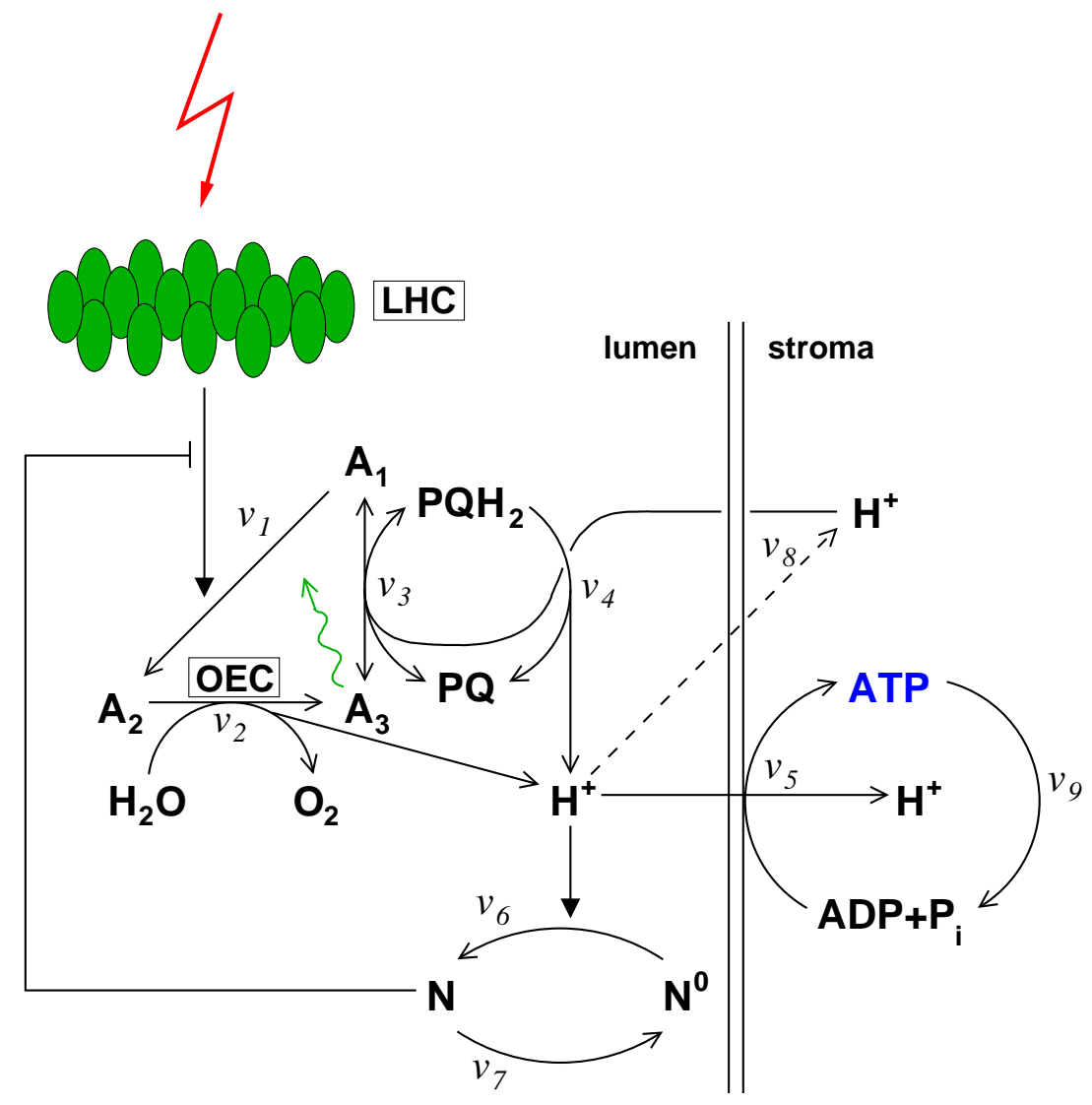

Figure 1: Schematic representation of the model. Light is absorbed by the light harvesting complexes (LHCs). Excitation energy is transferred to the open RCs $\left(A_{1}\right)$, leading to charge separation $\left(A_{2}\right)$. This process is inhibited by an active quencher $(N)$. The oxygen evolving complex (OEC) re-reduces the donor side of the $\mathrm{RC}$, resulting in state $A_{3}$ and the release of protons by water splitting. The acceptor side is re-oxidised by PQ, which receives protons from the stroma. $\mathrm{PQ}$ is re-oxidised by $\mathrm{Cytb}_{6} \mathrm{f}$, which is considered as external to the model and not shown in the figure. This re-oxidation involves proton release into the lumen. High proton concentration in the lumen triggers activation of a quencher $\left(N_{0}\right.$ and $N$ represent the inactive and active form, respectively). The proton gradient drives the production of ATP from ADP and inorganic phosphate $\left(\mathrm{P}_{\mathrm{i}}\right)$. ATP is consumed by external processes. State $A_{3}$ can be excited but not use the excitation energy for charge separation. The non-quenched fraction of this excitation energy is emitted as fluorescence (green arrow). The stoichiometries of the reactions are not shown.

separated charges. The rate $v_{1}$ of this process is described by Eq. (A.5) which takes into account that this process is ultimately driven by incoming 
light $\left(k_{1}\right)$ and inhibited by an active quencher $(N)$. During re-reduction of the electron donor $\left(v_{2}\right)$ by the oxygen evolving complex (OEC) one proton is released into the lumen per absorbed photon. The resulting state $A_{3}$ is re-oxidised on the acceptor side $\left(v_{3}\right)$ by electron transfer to plastoquinone (PQ), which receives protons from the stroma. The respective rates $v_{2}$ and $v_{3}$ are assumed to follow multilinear kinetics, the rate equations are given by Eqs. (A.6) and (A.7). In principle, recharging the donor and acceptor sides $\left(v_{2}\right.$ and $v_{3}$, respectively) may occur in opposite order, resulting in a four state description of the RCs. Such a four-state description of the RCs has been considered in Laisk et al. (1997). However, we found that for the parameters used here the additional state, in which only the acceptor side is re-oxidised, is almost not occupied and can therefore be neglected, leading to the present three-state description of the RCs.

Reduced PQ is re-oxidised by the cytochrome $\mathrm{b}_{6} \mathrm{f}\left(\mathrm{Cytb}_{6} \mathrm{f}\right)$ complex and re-oxidation is coupled with a release of protons into the lumen. For our minimal model, we draw the system boundary at this step and consider the $\mathrm{Cytb}_{6} \mathrm{f}$ pool as a constant, external quantity. This quantity can thus be included in the rate constant $k_{4}$ and the resulting linear rate law is given by Eq. (A.8).

Water splitting and PQ re-oxidation produces a proton gradient over the thylakoid membrane. The plastidic ATP synthase couples the resulting proton motive force to the biochemical synthesis of ATP from ADP and inorganic phosphate. For 14 protons which pass the membrane, 3 molecules of ATP are produced (Seelert et al., 2000). This coupling leads to a 'dynamic' equilibrium constant for the biochemical reaction which depends on the proton gradient and is determined by (see also Laisk et al. 2006)

$$
K_{e q}=e^{-\frac{\Delta G}{R T}} \quad \text { with } \quad \Delta G=\Delta G^{0}-\ln 10 \cdot \Delta \mathrm{pH} \cdot \frac{14}{3} \cdot R T,
$$

where $\Delta G^{0}$ is the standard change in Gibbs energy, $R$ the universal gas constant and $T$ the temperature. The $\Delta \mathrm{pH}$ dependent dynamic equilibrium is incorporated in a simple mass action kinetic rate law, leading to the ATP synthesis rate

$$
v_{5}=k_{5}\left([\mathrm{ADP}]-\frac{[\mathrm{ATP}]}{K_{e q}^{\prime}}\right), \quad \text { with } \quad K_{e q}^{\prime}=\left[\mathrm{P}_{\mathrm{i}}\right] \cdot K_{e q}
$$

where the inorganic phosphate concentration $\left[\mathrm{P}_{\mathrm{i}}\right]$ is assumed to be constant. 
Further, protons may passively leak into the stroma $\left(v_{8}\right)$. To balance the ATP production, we introduce one reaction $\left(v_{9}\right)$ representing all ATP consuming processes. These processes are assumed to follow mass action rate laws, see Eqs. (A.12) and (A.13).

An essential process associated with NPQ is the xanthophyll cycle. In this cycle, violaxanthin is deepoxidised in two steps to zeaxanthin by a luminally located deepoxidase, and the reverse epoxidation is catalysed by a stromal epoxidase. It has been shown (e.g., Pfündel and Dilley, 1993) that the deepoxidation reaction is strongly dependent on the luminal $\mathrm{pH}$, whereas the epoxidation reaction is independent (Gilmore et al., 1994). These findings are incorporated into the model by a simplified description of the xanthophyll cycle, in which a quencher can be either inactive or active (denoted $N_{0}$ and $N$, respectively) and the total amount of quencher is assumed to be constant and is normalised to unity, $N+N_{0}=1$. The quencher is assumed to be activated with the $\mathrm{pH}$-dependent rate

$$
v_{6}=k_{6}(1-N) \frac{\left[\mathrm{H}^{+}\right]^{n}}{\left[\mathrm{H}^{+}\right]^{n}+K_{Q}{ }^{n}} .
$$

This phenomenological Hill-type rate law is supported by experimental data by Pfündel and Dilley (1993), in which the rate of the deepoxidation reaction was determined for different $\mathrm{pH}$ values. An inflection $\mathrm{pH}$ of 6 (represented by the constant $K_{Q}$ ) was observed and a fit to the data yielded a Hill coefficient of $n=5.3$. For the inactivation rate $v_{7}$, a simple mass action law is assumed, see Eq. (A.11).

Besides the conserved quencher concentration (see above), three further conserved moieties restrict the system variables: The total number of RCs is denoted by $D$, the total (reduced and oxidised) PQ pool by $X$ and the adenosine phosphate pool in the stroma by $A^{\text {tot }}$.

\section{Results}

\subsection{Quasi steady-state approximation}

Processes within the RCs are much faster than NPQ induction. We thus apply a quasi steady-state approximation to the variables describing the states of the RCs $\left(A_{1}, A_{2}\right.$ and $\left.A_{3}\right)$ as well as the variable describing the oxidised fraction of the PQ pool $(P)$. For this, we consider the luminal proton concentration, $H$, and the activity of the quencher, $N$, as approximately 
constant on the time scale of the fast processes involving the excitation and closing of the RCs. Setting the rate equations of the fast variables to zero leads to a quadratic equation, dependent on light intensity and quenching activity, from which the oxidised fraction of the PQ pool can be determined as the positive root (see Eq. B.6 in Appendix B).

In the quasi steady-state approximation, the light driven proton influx into the lumen is proportional to the rate of $\mathrm{PQ}$ re-oxidation, which is given as $v_{4}=k_{4} \cdot(X-P)$, where $X-P$ is the concentration of reduced $\mathrm{PQ}$. Consequently, the quasi steady-state concentration of reduced PQ is decisive for the photosynthetic rate.

The overall excitation rate is given by the non-quenched fraction of the total excitation, $k_{1}(1-N)$. For the limit of large excitation rates, the PQ pool is maximally reduced. In this limit, the fraction of reduced PQ, denoted $q=(X-P) / X$, is given by

$$
q=\frac{k_{3}^{+} D}{k_{3}^{+} D+k_{4}} \approx 1
$$

where the approximation holds assuming that acceptor re-oxidation occurs significantly faster than $\mathrm{PQ}$ re-oxidation $\left(k_{3}^{+} D \gg k_{4}\right)$. In the opposite limit of no RC excitation, the PQ pool is completely oxidised $(q=0)$.

To study the influence of parameter values on the quasi steady-state, we systematically vary each parameter and compare the reduction state and resulting net proton import rate to a reference parameter set. The reference parameters have been derived from the literature and were subsequently fitted manually to experimental data (see Appendix C). In Fig. 2 the effects of the most influential parameters are depicted. While increasing the reoxidation rate, $k_{4}$, leads to a decreasing of the reduced PQ fraction (top left), the net proton influx (top right) is nevertheless increased. Interestingly, the reduction state of the $\mathrm{PQ}$ pool is mostly affected for low $\mathrm{RC}$ excitation rates while the proton influx changes for high rates. In low light, an increased re-oxidation will shift the balance of the PQ pool to more oxidised levels, however, the increased rate compensates the lower level of reduced PQ, resulting in unaltered net proton import rates. In high light, however, the PQ pool is maximally reduced regardless of the re-oxidation rate, explaining why an increased rate in this regime leads to higher proton import rates. Varying the total PQ pool has a very similar effect (centre row) and the explanation is analogous to that of changing the re-oxidation rate. Increasing the total number of RCs leads to a more reduced PQ pool. Concomitantly, the net 

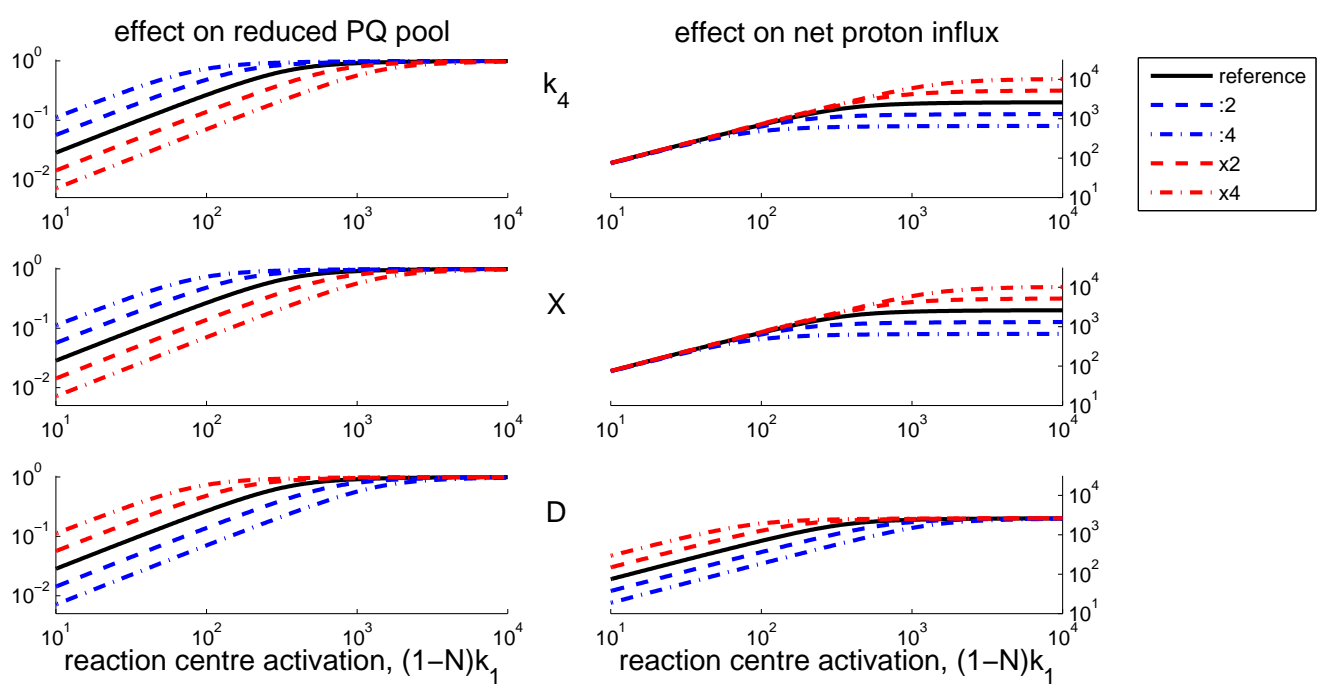

Figure 2: Effect of system parameters on the quasi steady-state of the reduced PQ pool (left column) and the net proton influx rate (right column). The three system parameters $k_{4}$ (PQ re-oxidation rate constant, top row), $X$ (total PQ pool, centre row) and $D$ (total RCs, bottom row) were varied and the effect of the quasi steady-state values were monitored. In each case, the values are shown in a double logarithmic plot as a function of RC activation rate, $(1-N) k_{1}$, for the reference parameters (black line), a two- and four-fold increase (red dashed and dash-dotted lines, respectively) and a two- and four-fold decrease of the corresponding parameter (blue dashed and dash-dotted lines, respectively). The parameter values used for the reference state are given in Table C.1.

proton import is also increased. However, this effect can only be observed for low $\mathrm{RC}$ excitation rates because under high light the $\mathrm{PQ}$ pool is maximally reduced irrespective of the numbers of RCs. The remaining parameters entering Eq. (B.6) only marginally influence the quasi steady-state reduction state and proton import rate, because water splitting and re-oxidation of the donor side of the RCs are assumed to occur orders of magnitudes faster than the other processes.

\subsection{Steady state photosynthesis}

Under constant light conditions, the photosynthetic rate will approach a stationary state which is determined by a balanced proton influx into the lumen and out-flux through the ATP synthase and proton leaks. Under steady-state conditions, the model equations can be simplified into a single 
equation depending only on one variable (the luminal free proton concentration, $H$ - see Appendix D), which can be numerically solved. In the model, the resulting $\mathrm{pH}$ critically depends on the proton accumulation due to the activity of the RCs, on ATP synthesis, external ATP consumption and on the stationary quenching activity. Based on results of Pfündel and Dilley (1993) on the $\mathrm{pH}$ dependence of the deepoxidation of violaxanthin and constant activity of the epoxidase (Gilmore et al., 1994), we assume a strong induction of quencher activation by low lumen $\mathrm{pH}$ and a constant deactivation rate. This leads to a sigmoid stationary quencher activity in dependence on lumen
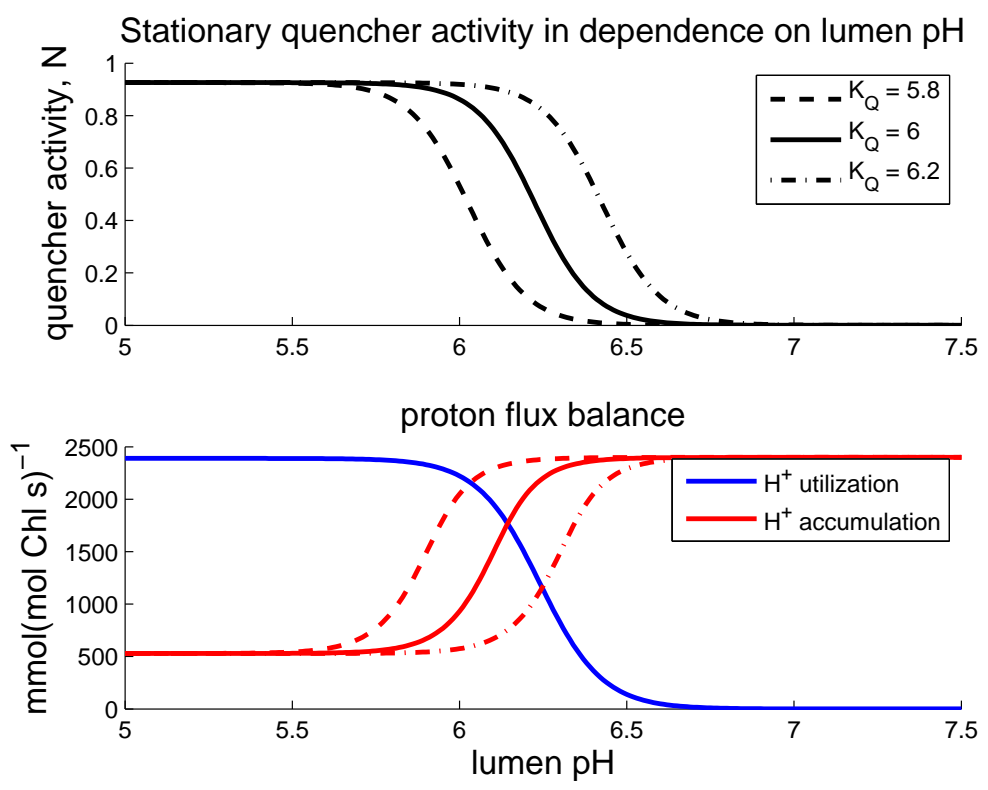

Figure 3: Stationary variables in dependence on lumen $\mathrm{pH}$. In the top panel, the quencher activity is depicted. The solid line represents the reference parameter set given in Table C.1. The dashed and dash-dotted lines indicate the shifted quencher activity as a result of a reduced $\left(K_{Q}=5.8\right)$ and increased $\left(K_{Q}=6.2\right)$ inflection $\mathrm{pH}$, respectively. The lower panel depicts the proton flux balance. The blue line represents the net proton utilisation by ATP synthase. These rates are independent on the inflection point $K_{Q}$. The red lines indicate the proton accumulation rate through the $\mathrm{RC}$ activity, including protons generated by the OEC and protons released in the lumen by PQ translocation (rates $v_{2}$ and $v_{4}$, respectively). Calculations were done for a constant light density $\left(800 \mu \mathrm{Em}^{-2} \mathrm{~s}^{-1}\right)$. The solid line has been calculated for the reference parameters, the dashed and dash-dotted lines again represent the behaviour for reduced $\left(K_{Q}=5.8\right)$ and increased $\left(K_{Q}=6.2\right)$ inflection $\mathrm{pH}$, respectively. 
$\mathrm{pH}$, with the inflection point $K_{Q}$ as a critical parameter determining the $\mathrm{pH}$ level required to induce quenching (see Fig. 3, top). How the inflection point determines the stationary lumen $\mathrm{pH}$ is illustrated by proton accumulation and utilisation rates in dependence on the $\mathrm{pH}$. In the lower panel of Fig. 3, the proton utilisation rate by ATP synthase activity is depicted in blue. The proton accumulation rate in the lumen as a result of $\mathrm{RC}$ activity (water splitting and proton translocation by $\mathrm{PQ}$ ) is depicted in red. Lower inflection $\mathrm{pH}$ $\left(K_{Q}\right)$ results in a lower quenching activation and therefore in higher proton accumulation rates in the lumen. The choice of $K_{Q}=6$ seems reasonable since a moderate drop in $\mathrm{pH}$ will result in quenching while simultaneously the stationary ATP synthase activity for moderate to strong light works with approximately $75 \%$ of its maximal activity.
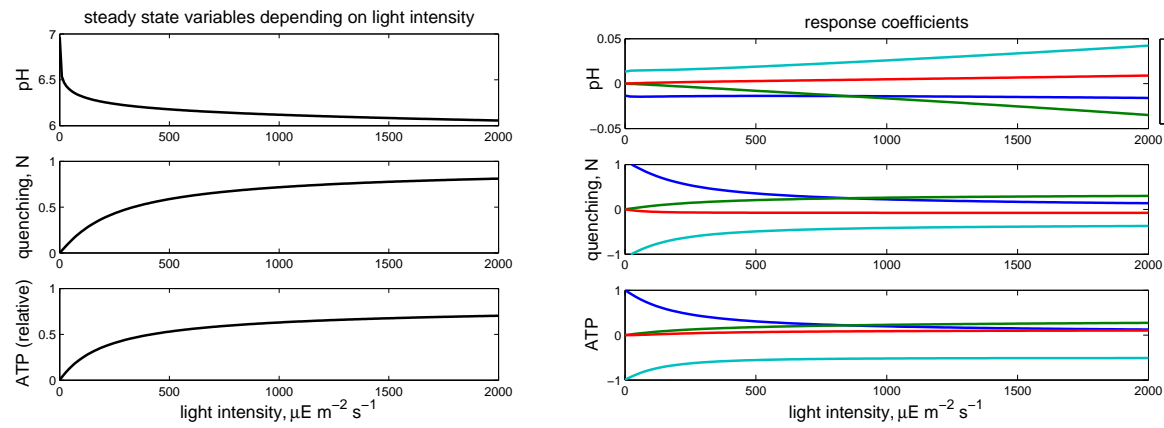

Figure 4: Steady state model behaviour for different light intensities. Left: The steady state variables lumen $\mathrm{pH}$, quenching activity and the ATP level as a fraction of the total adenosine phosphates are given in dependence on the photon flux density. Right: Response coefficients in dependence on the light intensity for the four critical system parameters total RCs $(D)$, total adenosine phosphate pool $(X)$, ATP synthase activity $\left(k_{5}\right)$ and external ATP consumption $\left(k_{9}\right)$. Calculations were performed with the reference parameters given in Table C.1.

In Fig. 4 (left) steady state lumen $\mathrm{pH}$, quenching activity and the ATP level relative to the total adenosine phosphate pool are shown as functions of photon flux density (PFD). A sharp drop in $\mathrm{pH}$ already for low light intensities can be observed. For higher intensities the $\mathrm{pH}$ level declines only moderately, in perfect accordance to experimental observations (Ruban et al., 2001). Quenching is induced already for low light intensities, however, it continues to increase for increased photon influx. Similarly, the stationary stromal ATP level increases with increasing light, however, the changes for 
high light conditions (above $1000 \mu \mathrm{Em}^{-2} \mathrm{~s}^{-1}$ ) are very moderate. The fact that for very low light an ATP level close to zero is predicted, is not in accordance with the observed nocturnal ATP:ADP ratio of around 1:2 (Heineke et al., 1991). This shortcoming can be explained by the simplifying model assumptions. The model includes ATP synthase as the only source of stromal ATP and, since in the absence of light this activity ceases, the model predicts zero ATP in the dark.

To study which parameters are most critically influencing the system's steady state behaviour, we systematically varied parameters and numerically recorded the changes on the steady state values. The strength of a parameter's influence is quantified by its response coefficient. For any system variable $y$ and parameter $p$, the normalised response coefficient is defined as

$$
R_{p}^{y}=\frac{p}{y} \frac{\partial y}{\partial p}=\frac{\partial \ln y}{\partial \ln p} .
$$

Naturally, the response coefficients depend on the reference state and thus on PFD. In Fig. 4 (right) four selected response coefficients are plotted as functions of the light intensity. An increased number of RCs $(D)$ has no significant influence on the steady-state lumen $\mathrm{pH}$, but - in particular for the low light regime - leads to increased quenching activity and a higher stromal ATP level. Increasing the total PQ pool $(X)$, on the other hand, results in changes predominantly in the high light regime, by leading to a decreased lumen $\mathrm{pH}$, increased quenching and increased ATP levels. Control of the maximal ATP synthase activity, $k_{5}$, is relatively small and the effects on quenching and ATP level are opposite. This behaviour is expected because a higher synthase activity promotes a faster proton consumption. Simultaneously, ATP production in the stroma is increased and thus, for unchanged consumption rate constants, the ATP level must increase. Critical control is exerted by the external ATP consumption process, $k_{9}$. A higher ATP requirement will lead to an increasing lumen $\mathrm{pH}$, and simultaneously strongly reduce quenching activity and stromal ATP levels. The latter result should be interpreted carefully since the assumption of linear kinetics for the overall ATP consumption is certainly an over-simplification. Metabolic regulation of the ATP demand is not included in the model and thus homoeostatic effects on the stationary ATP levels cannot be reproduced. 


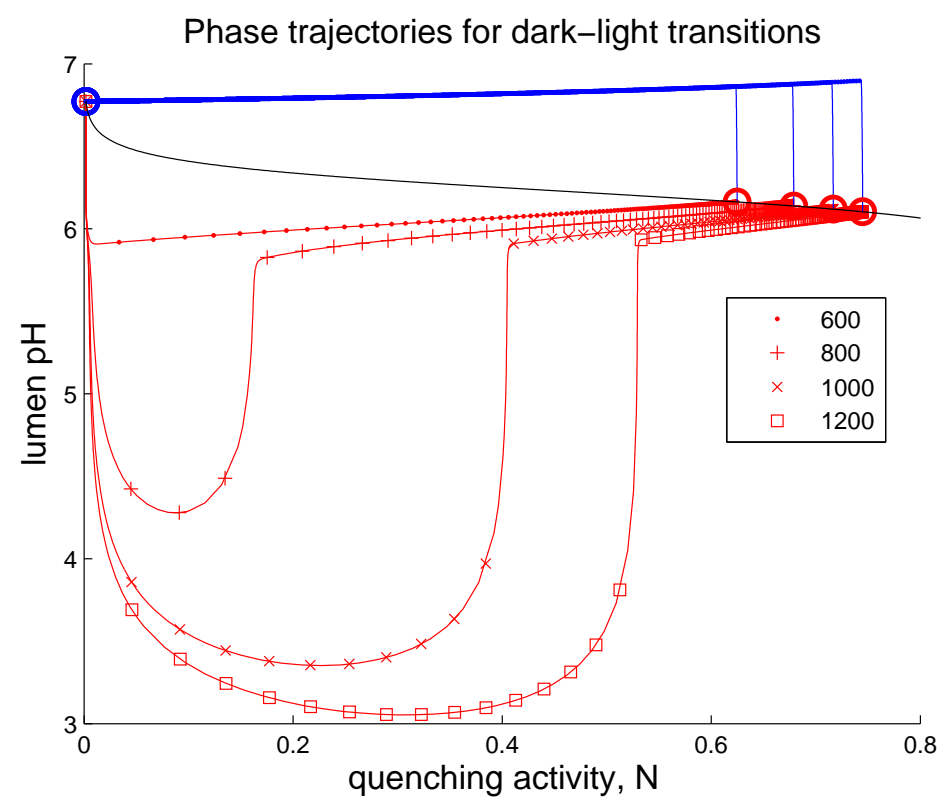

Figure 5: Trajectory of the two variables, luminal $\mathrm{pH}$ and quenching activity, during transitions from dark to light and vice versa. Shown are the trajectories for dark adapted systems (marked by the blue circle) upon sudden exposure to light of different intensities $\left(600,800,1000\right.$ and $1200 \mu \mathrm{Em}^{-2} \mathrm{~s}^{-1}$, red lines) and the trajectories for the reverse transition (blue lines) from the respective stationary states, marked by red circles. The markers on the trajectories are set in regular intervals of $1 \mathrm{~s}$ to visualise the different time scales on which the system is operating. The black line in the graph depicts the stationary states calculated for different light intensities.

\subsection{Light-dark transitions}

Next, we investigate how the model behaves under sudden changes in external light conditions. For this, simulations were started in the dark-adapted state and the transitions into light-adapted states for different PFDs were simulated. The phase plane trajectories of the quenching activity, $N$, and the lumen $\mathrm{pH}$ during the switch from dark to light and light to dark are depicted in Fig. 5. For all light intensities, an immediate decrease of $\mathrm{pH}$ is observed. Concomitantly, ATP synthase becomes active and stromal ATP levels rise (not shown in the plot). For light intensities above a threshold, $\mathrm{pH}$ levels drop further. Under such conditions, proton export by ATP synthase initially cannot balance proton accumulation. However, low $\mathrm{pH}$ triggers activation of the quencher resulting in a rapid alleviation of proton accumulation 
and the $\mathrm{pH}$ starts to rise within seconds. Quenching activation continues and the $\mathrm{pH}$ keeps rising on a slower time scale and the steady state value for the given light intensity is approximated. The reverse transition also displays two time scales. Without photon flux no protons are pumped into the lumen and $\mathrm{pH}$ begins to rise and ATP production ceases. With slower dynamics, the quenching agent is deactivated and eventually reaches its dark adapted steady state.
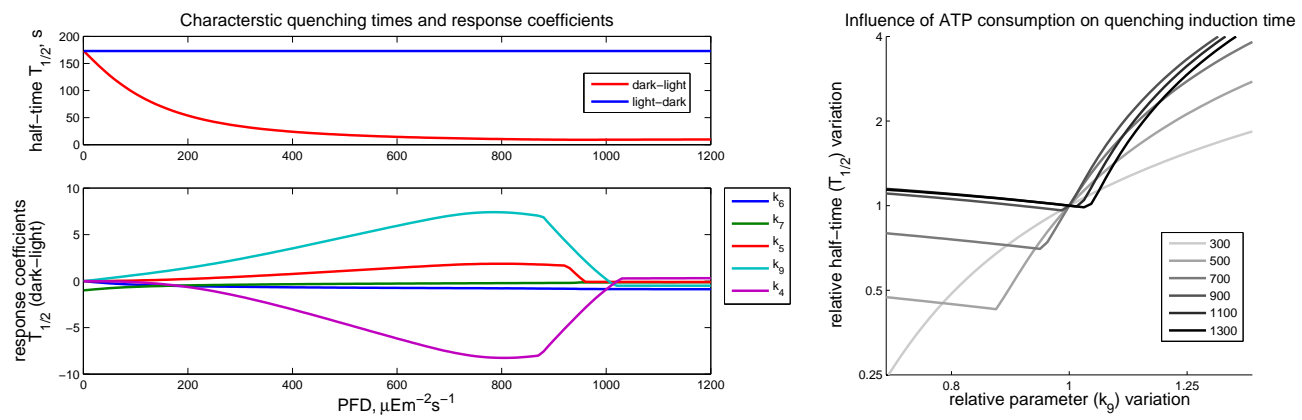

Figure 6: Quenching half-times and response coefficients for the quenching activation time for the most critical system parameters. In the top left panel, the activation (red) and deactivation (blue) half-times are shown as functions of the PFD of the induced light. For the same light intensities, the numerically determined control coefficients for the most influential parameters are depicted in the lower panel. The right graph depicts the responses of the half-times for non-infinitesimal changes of the parameter $k_{9}$ for the light intensities 300, 500, 700, 900, 1100 and $1300 \mu \mathrm{Em}^{-2} \mathrm{~S}^{-1}$ (light grey to black).

To study which system parameters determine the characteristic times of quenching activation, we determine the half-times, $T_{1 / 2}$, defined as the time the quenching variable $N$ requires to reach the mid-point between the dark and light adapted steady state values. In Fig. 6 (top left), the half-times for the activation and deactivation are plotted as functions of PFD. Deactivation dynamics under light-dark transition (blue line) are independent on the initial light intensity. The reason for this is that the only process included in the model promoting the deactivation (rate $v_{7}$ ) depends exclusively on the amount of active quencher. Interestingly, the transition from dark to light shows a strong dependence on light intensity. Dynamics are faster for higher photon flux density, which can be understood considering that stronger photon influx leads to a more rapid proton accumulation which in turn accelerates activation of the quencher. The control of the characteristic quenching 
activation time is complex. In Fig. 6 (bottom left), the response coefficients of the activation half-times for the most influential parameters are plotted as functions of the light intensity. The control of the parameters directly involved in quenching activation/deactivation ( $k_{6}$ and $k_{7}$, respectively) show an expected behaviour. An increase of either parameter will lead to a faster quenching activation. For low light intensities, the deactivation parameter and for high intensities the activation parameter dominates, reflecting the corresponding relative rates under the respective conditions. However, the rate parameters for reoxidation of the PQ pool $\left(k_{4}\right)$ and those related to ATP production and consumption ( $k_{5}$ and $k_{9}$, respectively) show an unexpected and highly unusual behaviour. The response coefficients depend strongly on the light intensity and display the largest absolute values for light intensities between 700 and $1000 \mu \mathrm{Em}^{-2} \mathrm{~s}^{-1}$. In this region, increasing external ATP consumption will for example lead to a drastic slow down of quenching activation. Since response coefficients are only local measures that characterise responses upon infinitesimal changes of the system parameters, this behaviour hints at non-linear responses for larger changes. To explore this interesting behaviour further, we exemplarily depict in Fig. 6 (right) the relative change in half-time as a function of the varied parameter $k_{9}$ for external ATP consumption for various light intensities. For a wide range of light intensities, the control of $k_{9}$ is bimodal in the sense that it exerts a small negative control on the half time below a critical threshold (slight negative slope in Fig. 6, right) and a strong positive control above this threshold. A closer investigation of the time courses of the quenching dynamics reveals that in the regime of low negative control the $\mathrm{pH}$ values temporarily drop significantly below their final stationary levels, analogous to the trajectories in Fig. 5 for light intensities of $800 \mu \mathrm{Em}^{-2} \mathrm{~s}^{-1}$ and above. Since under these conditions, quenching activation is maximal during the transient phase of low $\mathrm{pH}$, the half-time is predominantly determined by the final level of quenching activation. Since increased values of $k_{9}$ lead to reduced levels of stationary quenching activity (see Fig. 4, centre right), the mid point is reached faster, explaining the negative control on the half-time. Under conditions without significant transient decrease of $\mathrm{pH}$, the quenching dynamics are strongly $\mathrm{pH}$ dependent and thus largely determined by the stationary $\mathrm{pH}$. Since increased values of $k_{9}$ lead to increased stationary $\mathrm{pH}$ (see Fig. 4, top right), quenching dynamics will be considerably slower, thus explaining the strong positive control on the quenching half-time. 


\subsection{Comparison to experimental data}

Commonly used to study photosynthesis and NPQ experimentally is the so-called pulse-amplitude modulated (PAM) fluorescence technique. In these experiments, plant leaves (or algal suspensions) are usually dark adapted before the actual measurement and during the experiment the leaf is exposed to a weak (non-actinic) modulated measuring light, giving rise to the minimum $\left(F_{0}\right)$ chlorophyll fluorescence signal. Furthermore, the leaf is subjected to short saturating light pulses - transiently closing all RCs - giving rise to the maximum $\left(F_{M}\right)$ fluorescence. Additionally, actinic light of varying intensity and duration can be applied, inducing light adaptation processes in the photosynthetic apparatus, in particular NPQ. Quenching of qE-type results in excess excitation energy dissipation as heat and thus protects PSII from detrimental over-excitation.

Since chlorophyll fluorescence is not a system variable, it has to be calculated from the simulation output. If a chlorophyll molecule associated with a $\mathrm{RC}$ is excited, the excitation energy can be either used for charge separation (only in open RCs), emitted as light, leading to fluorescence, or dissipated as heat. In our model, states $A_{1}$ and $A_{3}$ can be excited and thus, can emit fluorescence. Fluorescence emanating from $A_{1}$ represents the amount of energy not used for charge separation and corresponds to the minimum chlorophyll fluorescence $\left(F_{0}\right)$ in the dark. We do not include $F_{0}$ in our calculation for the fluorescence signal because we do not resolve excitation and charge separation in the reaction $v_{1}$. Thus, only state $A_{3}$ is included in the calculation of the fluorescence signal. The activity of the quencher, $N$, determines the fraction of excitation energy emitted as heat, while the non-quenched fraction, $1-N$, is emitted as fluorescence. We therefore assume that the fluorescence signal is proportional to

$$
F=(1-N) \cdot \frac{A_{3}}{D} .
$$

Usually, PAM data are normalised to the highest measured fluorescence signal $\left(F_{M}\right)$ being set to 1 . Since the fluorescence signal defined by Eq. (6) is not capable to reflect the minimum chlorophyll fluorescence $\left(F_{0}\right)$ in the dark, we additionally normalise the lowest measured value to 0 . It has to be mentioned that of course $F_{0}$ is not 0 but reflects loss processes in the antenna system. To compare simulation to experimental data, we perform the same normalisation for the calculated fluorescence signal. In Fig. 7 the measured and simulated fluorescence signals are presented for three different light intensities $\left(300,600\right.$ and $\left.1200 \mu \mathrm{Em}^{-2} \mathrm{~s}^{-1}\right)$. It can be seen that the 


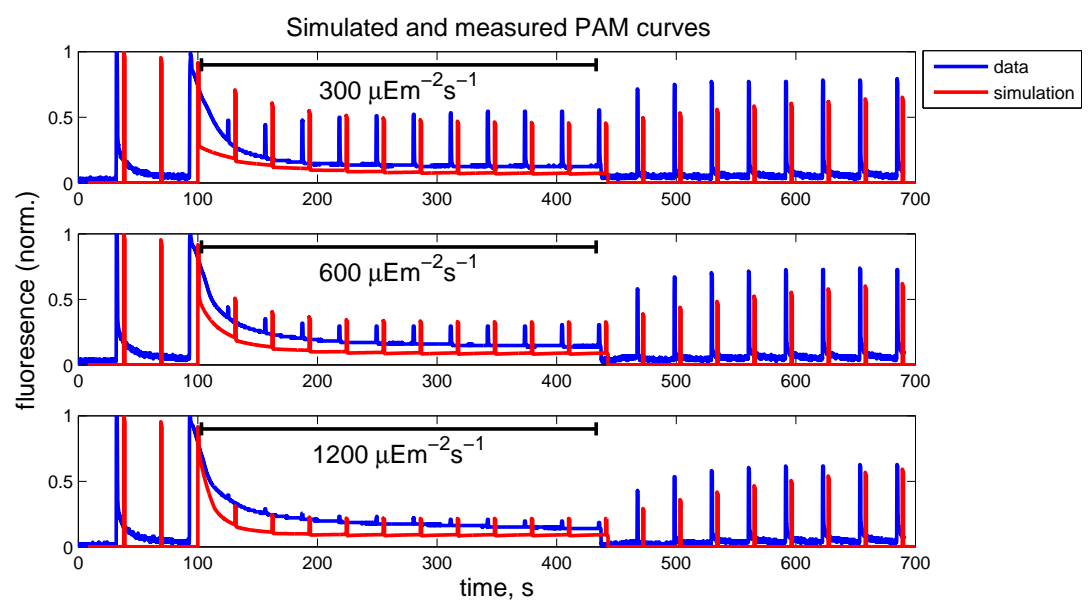

Figure 7: Experimental and simulated PAM curves. For the three actinic light intensities 300, 600 and $1200 \mu \mathrm{Em}^{-2} \mathrm{~s}^{-1}$, experimental data obtained from wild-type Arabidopsis thaliana leaves (blue lines) are shown together with the simulated fluorescence data. Experimental data was normalised such that the largest value equals 1 and the lowest observed value equals zero. Fluorescence was calculated from the simulated data as described in the text and the resulting values were normalised as the experimental data.

model reproduces the experimental data fairly well, at least qualitatively. For the moderate and high light intensities, the simulated light adaptation process reflects the experimental observations with high accuracy. However, for low light conditions, the experimental peak intensities during illumination show a slight increase after an initial drop. This behaviour is attributed to the fact that in vivo ATP synthase is inhibited in the dark and under low light intensities re-activation is slow. This process is not considered in our model and therefore an increase of fluorescence intensity during constant illumination cannot be reproduced. A further systematic discrepancy between simulation and data is observed after the light-dark transition. The simulated de-activation of the quenching activity is, under all light conditions, considerably slower than the experimentally observed relaxation dynamics.

\section{Discussion}

We have presented a highly simplified model of NPQ in PSII which serves the goals to a) investigate stationary photosynthetic rates, b) analyse NPQ induced adaptive dynamics of PSII upon changing light conditions, c) study 
the effects of variations of important pool sizes and reaction rate parameters and d) explore to what extent this simplistic approach is sufficient to reproduce experimental data obtained from PAM fluorescence measurements.

The simple model structure poses the advantage that many analytic formulas can be derived and dynamics can be thoroughly investigated. The general behaviour of the model is realistic. Simulated steady state observables lie in realistic ranges and the principle features of the dynamic quenching process are plausible. Under the transition from dark to light, two different dynamics are observed, depending on whether the lumen $\mathrm{pH}$ undergoes a transient drastic drop. Under low light conditions, proton accumulation can easily be balanced by ATP synthase activity and quenching induction is relatively slow. However, sudden exposure to high light leads to a situation where proton accumulation cannot be balanced by consumption through ATP synthase and quenching induction is faster, leading to feedback reduction of proton accumulation and proton flux is balanced within seconds.

Despite the general plausibility of the simulations, some systematic discrepancies to measured data are apparent. Most strikingly, the NPQ deactivation during the transition from light to dark is too slow in the simulation when compared to experimental PAM curves. The reason for this must be sought in the simplifying assumptions about the quenching process. We have assumed only a single quenching agent being directly related to zeaxanthin. However, it is well known that other factors play important roles (Horton et al., 2008). In particular, protonation induced conformational changes in the LHCs seem to contribute significantly to the qE-type quenching processes (Lokstein et al., 1994; Härtel et al., 1996; Lokstein et al., 2002). It is still remarkable that the highly simplified assumption that zeaxanthin directly acts as the sole quencher is sufficient for - at least - qualitative reproduction of the data, confirming a major role of zeaxanthin in $\mathrm{qE}$-type quenching. The fitted parameters for the quenching activation and deactivation processes $\left(k_{6}\right.$ and $k_{7}$, respectively) are about ten-fold higher than the measured rates for de-epoxidation and epoxidation (Pfündel and Dilley, 1993). This indicates that the assumption of zeaxanthin being the quencher $N$ (a variable in the range from 0 to 1 ) in the model is too simplistic. It rather supports the hypothesis that zeaxanthin is necessary for NPQ build-up but a few molecules of zeaxanthin are sufficient for maximum quenching (Härtel et al., 1996). This hypothesis could be tested by systematically exposing plants to different durations of high actinic illumination and subsequently recording the quenching relaxation dynamics, which should be slower for 
longer illumination times, due to higher levels of zeaxanthin accumulation.

Besides including such a saturation effect of zeaxanthin, the model can be extended to account for other $\mathrm{qE}$ factors, such as altered conformations of the LHCs. It will be interesting to compare the prediction of a more sophisticated model to PAM fluorometric data obtained for mutants in which the xanthophyll cycle is altered, such as the NPQ mutants of Arabidopsis thaliana (Lokstein et al., 2002).

In summary, our presented simplified model provides a useful theoretical description of PSII with which general dynamic properties of the photosynthetic light reactions and the adaptation of the photosynthetic apparatus to excess light can be studied. The model is easily extendable to include more mechanistic aspects of the qE-type quenching processes and may thus serve as a theoretical basis to accompany experimental approaches to explore the molecular basis of NPQ.

\section{Materials and Methods}

Chlorophyll fluorescence measurements were done with attached wildtype Arabidopsis thaliana leaves using a pulse-amplitude modulated (PAM) fluorometer FMS 2 (Hansatech) at ambient temperature as previously described (Lokstein et al., 2002).

\section{Funding}

This work was funded by the German Federal Ministry of Education and Research through the Systems Biology Research Initiative "GoFORSYS" (TH, HL, SS, KT) and by the Scottish Funding Council through the Scottish Universities Life Science Alliance, SULSA (OE).

\section{Appendix A. Model equations}

In the model represented in Fig. 1, water, oxygen, stromal protons and inorganic phosphate are considered external quantities and their concentration is held constant. The remaining variables $A_{1}, A_{2}$ and $A_{3}$ describe the RCs, $P$ and $Q$ quantify the oxidised and reduced PQ pool, respectively, $H$ the proton concentration in the lumen, $N^{0}$ and $N$ the inactive and active quencher, respectively, and $S$ and $T$ describe the stromal ADP and ATP 
concentration respectively. These variables are restricted by the conserved quantities

$$
\begin{aligned}
\text { conserved number of RCs: } & A_{1}+A_{2}+A_{3}=D \\
\text { conserved PQ pool : } & P+Q=X \\
\text { conserved adenosine phosphate pool : } & S+T=A^{\text {tot }} \\
\text { conserved quencher : } & N^{0}+N=1 .
\end{aligned}
$$

Choosing $A_{1}, A_{2}, P, H, N$ and $T$ as independent variables, the rate expressions read

$$
\begin{aligned}
v_{1}= & (1-N) \cdot k_{1} \cdot A_{1} \\
v_{2}= & k_{2} \cdot A_{2} \\
v_{3}= & k_{3}^{+} \cdot\left(D-A_{1}-A_{2}\right) \cdot P-k_{3}^{-} A_{1} \cdot(X-P) \\
v_{4}= & k_{4} \cdot(X-P) \\
v_{5}= & k_{5}\left(A^{\text {tot }}-T\left(1+\frac{1}{K_{e q}{ }^{\prime}(H)}\right)\right) \\
& \text { where } K_{e q}{ }^{\prime}(H)=\left[\mathrm{P}_{\mathrm{i}}\right] \cdot e^{-\frac{\Delta G^{0}}{R T}} \cdot\left(\frac{\left[\mathrm{H}^{+}\right]_{\text {stroma }}}{H}\right)^{\frac{14}{3}} \\
v_{6}= & k_{6}(1-N) \frac{H^{n}}{H^{n}+K_{Q}{ }^{n}} \\
v_{7}= & k_{7} \cdot N \\
v_{8}= & k_{8} \cdot\left(H-\left[\mathrm{H}^{+}\right]_{\text {stroma }}\right) \\
v_{9}= & k_{9} \cdot T .
\end{aligned}
$$

The rate $k_{1}$ is dependent on the photon influx and determines the external light input of the system. To convert PFD into an $\mathrm{RC}$ activation rate, we assume

$$
k_{1}=c_{P F D} \cdot P F D
$$

where PFD is the PFD measured in $\mu \mathrm{Em}^{-2} \mathrm{~s}^{-1}$ and $c_{P F D}$ is a manually fitted conversion factor. The differential equations for the six free variables read

$$
\begin{aligned}
\dot{A_{1}} & =v_{3}-v_{1} \\
\dot{A_{2}} & =v_{1}-v_{2} \\
\dot{P} & =v_{4}-v_{3}
\end{aligned}
$$




$$
\begin{aligned}
\dot{H} & =b_{H}\left(2 v_{2}+v_{4}-\frac{14}{3} v_{5}-v_{8}\right) \\
\dot{N} & =v_{6}-v_{7} \\
\dot{T} & =v_{5}-v_{9} .
\end{aligned}
$$

For Eq. (A.18) determining the rate of change of the free proton concentration, we assume that protons are highly buffered in the lumen and, following Laisk et al. (2006) we apply a simple proportionality between the total number of protons and those contributing to the free proton concentration by introducing the buffering constant $b_{H}$.

\section{Appendix B. Quasi steady-state approximation}

Transitions and electron transport within the RCs is considerably faster than the time scales on which quenching processes are observed. Thus, rates $v_{1}, v_{2}$ and $v_{3}$ are orders of magnitude faster than the other reaction rates. We therefore assume that the variables $A_{1}, A_{2}$, and $P$ are approximately in steady state when considering the slower time scale of the other variables and set Eqs.(A.15)-(A.17) to zero,

$$
\begin{aligned}
& v_{3}-v_{1}=k_{3}^{+}\left(D-A_{1}-A_{2}\right) P-k_{3}^{-} A_{1}(X-P)-f k_{1} A_{1}=0 \\
& v_{1}-v_{2}=f k_{1} A_{1}-k_{2} A_{2}=0 \\
& v_{3}-v_{4}=k_{3}^{+}\left(D-A_{1}-A_{2}\right) P-k_{3}^{-} A_{1}(X-P)-k_{4}(X-P)=0
\end{aligned}
$$

where $f=1-N$ has been introduced. Eq. (B.2) implies

$$
A_{2}=\frac{f k_{1}}{k_{2}} A_{1}
$$

and subtracting Eq. (B.3) from Eq. (B.1) yields

$$
A_{1}=\frac{k_{4}}{f k_{1}}(X-P) \text {. }
$$

Eliminating $A_{1}$ and $A_{2}$ from Eq. (B.3) yields the quadratic equation

$$
P^{2}(\alpha-\beta)+P\left(k_{3}^{+} D-\alpha X+2 \beta X+k_{4}\right)-\beta X^{2}-k_{4} X=0,
$$

where the abbreviations

$$
\alpha=k_{3}^{+} k_{4}\left(\frac{1}{f k_{1}}+\frac{1}{k_{2}}\right) \quad \text { and } \quad \beta=\frac{k_{3}^{-} k_{4}}{f k_{1}}
$$


have been introduced. Eq. (B.6) together with Eqs. (B.5) and (B.4) allows to determine the oxidised $\mathrm{PQ}$ and the states of the RCs in dependence on the light influx $\left(k_{1}\right)$ and the quenching status $(f=1-N)$. The condition $k_{3}^{+}>k_{3}^{-}$, which is certainly fulfilled because $\mathrm{PQ}$ reduction is energetically driven by the activated RCs, ensures that Eq. (B.6) has exactly one positive root $(\alpha-\beta>0$ and the left hand side of Eq. (B.6) is negative for $P=0$ ). The uniqueness of the solution guaranteed allows to define a function

$$
P=P\left(k_{1}, N\right)
$$

by which the model equations can be reduced to a three variable system, governed by Eqs. (A.18)-(A.20),

$$
\begin{aligned}
\frac{1}{b_{H}} \dot{H}= & 3 k_{4}\left(X-P\left(k_{1}, N\right)\right)-\frac{14}{3} k_{5}\left(A^{\text {tot }}-T\left(1+\frac{1}{K_{e q}{ }^{\prime}(H)}\right)\right) \\
& -k_{8}\left(H-\left[\mathrm{H}^{+}\right]_{\text {stroma }}\right) \\
\dot{N}= & k_{6}(1-N) \frac{H^{n}}{H^{n}+K_{Q}{ }^{n}}-k_{7} N \\
\dot{T}= & k_{5}\left(A^{\text {tot }}-T\left(1+\frac{1}{K_{e q}^{\prime}(H)}\right)\right)-k_{9} T
\end{aligned}
$$

\section{Appendix C. Choice of Parameters}

The parameters chosen for the reference state are given in Table C.1. Several parameters are available from literature resources. Where applicable, the corresponding sources are given in the last column. The units of concentration chosen for the system variables are $\operatorname{mmol}(\mathrm{mol} \mathrm{Chl})^{-1}$. The conversion from standard concentration units were performed using values from Laisk et al. (2006), who assumed a Chlorophyll content of $350 \cdot 10^{-6} \mathrm{~mol}$ per $\mathrm{m}^{2}$ thylakoid membrane and lumen and stroma volumes of $0.0014 \mathrm{l} \mathrm{m}^{-2}$ and $0.0112 \mathrm{l} \mathrm{m}^{-2}$, respectively. Thus, $1 \mathrm{mmol}(\mathrm{mol} \mathrm{Chl})^{-1}$ corresponds to $2.5 \cdot 10^{-4} \mathrm{M}$ in the lumen and $3.2 \cdot 10^{-5} \mathrm{M}$ in the stroma. The plastoquinone re-oxidation rate constant $\left(k_{4}\right)$ and the ATP synthesis and external ATP consumption rate constants ( $k_{5}$ and $k_{9}$, respectively) were manually fitted within reasonable ranges to reflect realistic stationary ATP:ADP ratios for a wide range of light conditions. The quenching activation and deactivation rate constants ( $k_{6}$ and $k_{7}$, respectively) were first obtained from the literature and then manually fitted to reflect experimentally obtained quenching 
Table C.1: Rate Parameters used throughout the paper as a reference.

\begin{tabular}{|c|c|c|}
\hline parameter & value & reference/comment \\
\hline$k_{2}$ & $3.4 \cdot 10^{6} \mathrm{~s}^{-1}$ & Sonneveld et al. (1979) \\
\hline$k_{3}^{+}$ & $1.56 \cdot 10^{5} \mathrm{mmol}(\mathrm{mol} \mathrm{Chl} \mathrm{s})^{-1}$ & Haehnel (1976) \\
\hline$k_{3}^{-}$ & $3.12 \cdot 10^{4} \mathrm{mmol}(\mathrm{mol} \mathrm{Chl} \mathrm{s})^{-1}$ & Lebedeva et al. (2002) \\
\hline$k_{4}^{3}$ & $50 \mathrm{~s}^{-1}$ & fitted \\
\hline$k_{5}$ & $80 \mathrm{~s}^{-1}$ & Pänke and Rumberg (1996) \\
\hline$k_{6}$ & $0.05 \mathrm{~s}^{-1}$ & Pfündel and Dilley (1993), fitted \\
\hline$k_{7}$ & $0.004 \mathrm{~s}^{-1}$ & Pfündel and Dilley (1993), fitted \\
\hline$k_{8}$ & $10 \mathrm{~s}^{-1}$ & estimated \\
\hline$k_{9}$ & $20 \mathrm{~s}^{-1}$ & fitted \\
\hline$D$ & $2.5 \mathrm{mmol}(\mathrm{mol} \mathrm{Chl})^{-1}$ & Schöttler et al. (2004) \\
\hline$X$ & $17.5 \mathrm{mmol}(\mathrm{mol} \mathrm{Chl})^{-1}$ & Kirchhoff et al. (2002) \\
\hline$A$ & $32 \mathrm{mmol}(\mathrm{mol} \mathrm{Chl})^{-1}$ & Usuda (1988) \\
\hline$K_{Q}$ & $0.004 \mathrm{mmol}(\mathrm{mol} \mathrm{Chl})^{-1}$ & corresponds to $\mathrm{pH} 6$ \\
\hline$n$ & 5 & Pfündel and Dilley (1993) \\
\hline$b_{H}$ & 0.01 & Laisk et al. (2006) \\
\hline$c_{P F D}$ & $4 / 3$ & fitted \\
\hline$\Delta G^{0}$ & $30.6 \mathrm{~kJ}(\mathrm{~mol})^{-1}$ & Rosing and Slater (1972) \\
\hline$\left[\mathrm{P}_{\mathrm{i}}\right]$ & $10 \mathrm{mM}$ & Usuda (1988) \\
\hline$\left[\mathrm{H}^{+}\right]_{\text {stroma }}$ & $6.34 \cdot 10^{-5} \mathrm{mmol}(\mathrm{mol} \mathrm{Chl})^{-1}$ & corresponds to $\mathrm{pH} 7.8$ \\
\hline$R$ & $0.0083 \mathrm{~kJ}(\mathrm{~mol} \mathrm{~K})^{-1}$ & universal gas constant \\
\hline$T$ & $298 \mathrm{~K}$ & corresponds to $25^{\circ} \mathrm{C}$ \\
\hline
\end{tabular}

dynamics. The free parameter $c_{P F D}$, which translates the PFD in units of $\mu \mathrm{Em}^{-2} \mathrm{~s}^{-1}$ into an $\mathrm{RC}$ activation rate in $\mathrm{mmol}(\mathrm{mol} \mathrm{Chl} \mathrm{s})^{-1}$ was finally fitted to reflect the experimental curves obtained for the different light intensities.

\section{Appendix D. Steady state solution}

Under constant light conditions, the system will assume a stationary state which is determined by $\dot{H}=\dot{N}=\dot{T}=0$. Following Eqs. (B.10 and B.11), the stationary values of $N$ and $T$ can be expressed as functions of $H$ :

$$
\begin{aligned}
& N(H)=\frac{1}{1+\frac{k_{7}}{k_{6}} \frac{H^{n}+K_{Q}^{n}}{H^{n}}}, \\
& T(H)=\frac{A^{\text {tot }}}{1+\frac{1}{K_{e q}{ }^{\prime}(H)}+\frac{k_{5}}{k_{9}}} .
\end{aligned}
$$

These expressions allow to eliminate $T$ and $N$ from the steady state condition $\dot{H}=0$, resulting in an equation which only depends on $H$. Solving this equation numerically yields the steady state $\mathrm{pH}$ level for constant light influx, 
from which the other steady state variables may be determined by Eqs. (D.1) and (D.2).

\section{References}

Frank, H., Cua, A., Chynwat, V., Joung, A., Gosztola, D., Wasielewski, M., 1994. Photophysics of the carotenoids associated with the xanthophyll cycle in photosynthesis. Photosynth Res 41, 389-395.

Gilmore, A.M., Mohanty, N., Yamamoto, H.Y., 1994. Epoxidation of zeaxanthin and antheraxanthin reverses non-photochemical quenching of photosystem II chlorophyll a fluorescence in the presence of trans-thylakoid delta pH. FEBS Lett 350, 271-274.

Haehnel, W., 1976. The reduction kinetics of chlorophyll aI as an indicator for proton uptake between the light reactions in chloroplasts. Biochim Biophys Acta 440, 506-521.

Härtel, H., Lokstein, H., Grimm, B., Rank, B., 1996. Kinetic studies on the xanthophyll cycle in barley leaves (influence of antenna size and relations to nonphotochemical chlorophyll fluorescence quenching). Plant Physiol 110, 471-482.

Heineke, D., Riens, B., Grosse, H., Hoferichter, P., Peter, U., Flügge, U.I., Heldt, H.W., 1991. Redox transfer across the inner chloroplast envelope membrane. Plant Physiol 95, 1131-1137.

Horton, P., Johnson, M.P., Pérez-Bueno, M.L., Kiss, A.Z., Ruban, A.V., 2008. Photosynthetic acclimation: does the dynamic structure and macroorganisation of photosystem II in higher plant grana membranes regulate light harvesting states? FEBS J 275, 1069-1079.

Kirchhoff, H., Mukherjee, U., Galla, H.J., 2002. Molecular architecture of the thylakoid membrane: lipid diffusion space for plastoquinone. Biochemistry 41, 4872-4882.

Laisk, A., Eichelmann, H., Oja, V., 2006. C3 photosynthesis in silico. Photosynth Res 90, 45-66. 
Laisk, A., Oja, V., Rasulov, B., Eichelmann, H., Sumberg, A., 1997. Quantum yields and rate constants of photochemical and nonphotochemical excitation quenching (experiment and model). Plant Physiol 115, 803-815.

Lebedeva, G.V., Beliaeva, N.E., Demin, O.V., Riznichenko, G.I., Rubin, A.B., 2002. [kinetic model of primary processes of photosynthesis in chloroplasts. fast phase of chlorophyll fluorescence induction under light of various intensity]. Biofizika 47, 1044-1058.

Lokstein, H., Härtel, H., Hoffmann, P., Woitke, P., Renger, G., 1994. The role of light-harvesting complex II in excess excitation energy dissipation: an in-vivo fluorescence study on the origin of high-energy quenching. J. Photochem. Photobiol. B: Biol. 26, 175-184.

Lokstein, H., Tian, L., Polle, J.E.W., Della Penna, D., 2002. Xanthophyll biosynthetic mutants of Arabidopsis thaliana: altered nonphotochemical quenching of chlorophyll fluorescence is due to changes in Photosystem II antenna size and stability. Biochim Biophys Acta 1553, 309-319.

Pänke, O., Rumberg, B., 1996. Kinetic modelling of the proton translocating $\mathrm{CF}_{0} \mathrm{CF}_{1}$-ATP synthase from spinach. FEBS Lett 383, 196-200.

Pfündel, E.E., Dilley, R.A., 1993. The pH dependence of violaxanthin deepoxidation in isolated pea chloroplasts. Plant Physiol 101, 65-71.

Quick, W., Stitt, M., 1989. An examination of factors contributing to non-photochemical quenching of chlorophyll fluorescence in barley leaves. Biochim Biophys Acta 977, 287-296.

Rosing, J., Slater, E.C., 1972. The value of G degrees for the hydrolysis of ATP. Biochim Biophys Acta 267, 275-290.

Ruban, A.V., Wentworth, M., Horton, P., 2001. Kinetic analysis of nonphotochemical quenching of chlorophyll fluorescence. 1. Isolated chloroplasts. Biochemistry 40, 9896-9901.

Schöttler, M.A., Kirchhoff, H., Weis, E., 2004. The role of plastocyanin in the adjustment of the photosynthetic electron transport to the carbon metabolism in tobacco. Plant Physiol 136, 4265-4274. 
Seelert, H., Poetsch, A., Dencher, N.A., Engel, A., Stahlberg, H., Müller, D.J., 2000. Structural biology. Proton-powered turbine of a plant motor. Nature 405, 418-419.

Sonneveld, A., Rademaker, H., Duysens, L.N., 1979. Chlorophyll a fluorescence as a monitor of nanosecond reduction of the photooxidized primary donor P-680 of photosystem II. Biochim Biophys Acta 548, 536-551.

Thornley, J.H.M., 1974. Light fluctuations and photosynthesis. Ann Bot 38, 363-373.

Usuda, H., 1988. Adenine nucleotide levels, the redox state of the NADP system, and assimilatory force in nonaqueously purified mesophyll chloroplasts from maize leaves under different light intensities. Plant Physiol 88, 1461-1468.

Vredenberg, W.J., 2009. Kinetic models of photosystem II should accommodate the effect of donor side quenching on variable chlorophyll A fluorescence in the microseconds time range. Photosynth Res 102, 99-101.

Zhu, X.G., Govindjee, Baker, N.R., de Sturler, E., Ort, D.O., Long, S.P., 2005. Chlorophyll a fluorescence induction kinetics in leaves predicted from a model describing each discrete step of excitation energy and electron transfer associated with Photosystem II. Planta 223, 114-133. 